\title{
Organization design of complex technical products in integrated information systems
}

\author{
Evgeny Dubrovsky*, and Viktor Dmitriev \\ Tomsk Polytechnic University, 634050 Tomsk, Russian Federation
}

\begin{abstract}
The article substantiates the necessity of using modern tools for the design of advanced space technology. The problems and consequences are presented in the application of CAD of various profiles within the framework of a single technical project. The decision of problems is resulted. The description of the through design process and the integrated information system is given. The possibilities of integrated information systems and the expected business effect are shown in their implementation and organization of an end-to-end design process.
\end{abstract}

\section{Introduction}

Engineering of spacecraft is complex and hi-tech process. The most part of highly qualified engineers are working on the design of spacecraft. In variety of industries the market sees a lot of change happening these days; new trends and challenges obviously need to be addressed as part of the product creation process [1]. Engineering design is a source of competitive advantage for manufacturing companies [2-8]. Manufacturing finished products and testing engineers using special CAD $\backslash \mathrm{CAM} \backslash \mathrm{CAE}$ systems are preparing production for design and modelling [9]. CAD $\backslash \mathrm{CAM} \backslash \mathrm{CAE}$ systems allow to gain more speed in project development, and to reduce duration of development new spacecraft's, and to improve quality development because of speed and reliability of computer technology, reliability of mathematical models, effective methods of optimization, and exclusion of the human factor. Use of CAD $\backslash$ CAM $\backslash C A E$ systems allows more qualitatively to solving questions related to:

- A large amount of work on the design and modeling of products;

- Time and financial constraints in the design;

- The need for a detailed engineering of the spacecraft as a whole;

- The need to organize stage-by-stage control of the design and manufacture of the finished product.

\section{CADICAMICAE for industry}

In this article CAD $\backslash \mathrm{CAM} \backslash \mathrm{CAE}$ systems widely used in industry are considered. The most important points are:

*Corresponding author: evgenij.dubrovskij@gmail.com 
- ECAD - system for electrical design;

- MCAD - system for part and product design.

\subsection{ECAD and MCAD}

Between ECAD and MCAD systems, there are cardinal differences in the functional purpose and data representation, but integrated using of ECAD and MCAD systems allows quick and qualitative problem solving:

- Preparation of input data;

- Electric preparation of production;

- Design preparation of production;

- Technological preparation of production.

Engineering of spacecraft requires a well-developed end-to-end design technology, where the design process should be closely related, and ECAD and MCAD integrated into a single information system. Let us explain in more detail.

\subsection{Problems with integration}

The design process of a complex technical product is divided into electrical and design parts, in order to meet both part's requirements, integrated use of the MCAD and ECAD systems in the same project is highly recommended. Usually data's getting from ECAD system (electrical circuits) are input data for MCAD systems. For example, block layout design, product design and cables network topology are made with using of data obtained from the development results of ECAD product schematic diagrams. At the same time, the task of electrical design is to organize the correct connection of wires, connectors, cables and the organization of the required signal transmission between the equipment. In turn, the task of designing the product design is to organize the maximum compactness of the arrangement of devices and the optimal topology of the cable network.

Designing a product by using of two or more different CAD systems imposes necessity of data exchange between them. That, in turn, requires development or implementation of special software with subsystems that ensure mutual integration, because the problem is that data cannot always be transferred between CAD without preliminary conversion. Converting data from one format to other often results in the accumulation of errors in the design process. Errors during design process will cause negative influence to design results, and it can be detected only during manufacturing, installation or testing process, what is absolutely unacceptable.

On practice, the problem is complicated by the fact that within a single project, specialists can use several ECAD systems that do not have direct data exchange with a 3D MCAD system.

Consequence of the lack of integration between ECAD and MCAD systems is the often inconsistencies between the electrical circuits and design of product. If there is an error in the electrical circuit or the designer can not implement the circuit design, there is no way to correct quickly the situation. Often it is necessary to repeat the stage of electrical design again, or to spent time for revision, which is by no means fast. In addition, during the revision process construction design information can become out of date. As the result, it is a great loss of time. Solving of this conflict situations is directly depends on human factor and it is not always the result of objective analysis, so it can't leads to the optimal decision making. 


\section{Solutions}

Engineers from different countries work over solution of described problems. Today, the most effective, universally recognized and used in the world practice is the organization of end-to-end design process at enterprises and development or implementation of integrated information systems instead of individual CAD systems.

The integrated approach is able to design, maintain and produce other data, process and metadata management in the database, monitoring its development [3]. The end-to-end process is continuous sequence of actions, where each next stage must be based on the results of previous stage. At the same time, it must be ensured that the operations are carried out not only in a directly, but also in the opposite direction, which makes it possible to solve problems more effectively:

- Return of the received input data for revision of dimensional parameters;

- Operative finding of errors in the circuit solution;

- Revision of the electrical circuit;

- Ensuring maximum control over the life cycle of the product;

- Exclude the possibility of loss of design data;

- To ensure the security of data storage.

Integrated information systems represent the software for designing, built into a single information space based on a single software platform. In the framework of software platform, data can be directly exchanged between components, ECAD and MCAD, which must have good compatibility in all technological design operations.

Because of the fulfillment of this condition, the integrated information systems allow completely:

- To organize end-to-end design technology in practice;

- Maximize the design process;

- To differentiate the rights of access of developers;

- Reduce the costs of design, manufacture and testing;

- Reduce of errors the human factor, due to automation;

- Organize work in a unified system of accounting, control and storage of data;

- Promotes the organization of paperless design technology.

\section{Conclusions}

The organization of an end-to-end design and implementation of integrated information systems leads to:

- improving the quality of products;

- reducing the cost of products;

- Reducing time to market.

As a result, the solution of these problems leads to the main goal of the enterprise - to increase the competitiveness of the enterprise's products on the world market.

\section{References}

1. A. Barth, G.L. Kovács, D. Kochan, IFIP AICT 411 (2013)

2. T. Boudouh, S. Gomes, MATEC Web of Conferences 112 (2017)

3. M. G. Trotta, Journal of Industrial Engineering and Management (2010)

4. V. S Dmitriev, G. N. Gladushev, T. G.Kostuchenko, KORUS 23, (2001)

5. R. C. W. Sung, J. M. Ritchie, T. Lim, Z. Kosmadoudi, Virtual Reality (2012) 
6. B. L. Wan, W. X. He, C. Y. Chen, MATEC Web of Conferences 44, (2016)

7. A. V. Taracenko, V. S. Dmitriev, T. G.Kostuchenko, KORUS 83, (2005)

8. Yu. Britova, V. Dmitriev, T. Kostyuchenko, IOP Conference Series: Materials Science and Engineering 132(1), (2016)

9. V. E. Baranova, P. F. Baranov, Dynamics of Systems, Mechanisms and Machines (2014) 